\title{
AbleProfessionals: A Recruiting and Accommodation Service for Atlanta Employers
}

\author{
by \\ J. Hunter Ramseur, M Ed., C.R.C., and \\ Lee S. Gardner, M.S.; and Darcy S. Painter \\ Center for Rehabilitation Technology, Georgia Institute of Technology, \\ Atlanta, Georgia, USA
}

\begin{abstract}
In the 1990s and beyond, businesses face increasing demands for a competitive edge in the global marketplace. Nations also face similar global economic pressures. It is clear that effective, efficient use of human and technological resources are key factors for national economic survival as we enter the next millennium. One measure of human resource efficiency in a society is the percentage of its members who contribute to the economy. The society's technological resources, in the form of accessible computer tools integrated into the workplace, can enable the contribution of a larger percentage of its members. This paper presents a business being started in Atlanta, Georgia, USA, that assists corporations in hiring people with disabilities by offering to locate and recruit quality candidates and provide complete, cost effective accommodation service including design, and installation of office technology to enable them to be effectively employed.
\end{abstract}

\section{The Shortcomings of Traditional Hiring Processes}

When corporations or businesses decide to do the right thing and hire a person with a disability, they often are penalized. This is because the traditional approach to hiring people into a corporation is based on assumptions about the average person that are violated when the issue of hiring a person with a disability is considered. The problems that the recruitment and accommodation of people with disabilities pose for traditional human resource organizations can be costly and complex. To hire a person with a disability corporations must frequently go to great lengths to locate and recruit their perspective employee as well as coordinate any accommodations that are necessary in computer equipment, office furniture or building architecture. These activities drive up the cost of hiring a person with a disability and add a significant technical challenge to the process in the form of the computer tools and office accessibility issues that must be addressed if people with disabilities are to be able to work. These factors combine to create the perception that hiring a person with a disability is too much additional work and is too risky. Doing the right thing is deemed too complicated, too expensive and the legal risks are too great. The end result is that very few people with disabilities are hired by employers.

For corporations to offer people with disabilities opportunities to work commensurate with their qualifications and potential, the problems of technical expertise, proper equipment and accommodations must be solved. The cost and time investments on the 
part of the corporate employer to hire people with disabilities must come closer to the well known, commonly accepted costs of hiring traditional employees.

\section{The AbleProfessionals Solution}

The Center for Rehabilitation Technology, Inc. (CRT, Inc.), at the Georgia Institute of Technology in Atlanta, Georgia has devised a practical solution to the problems of locating, recruiting and arranging the computer accommodations of people with disabilities. This new business venture is called AbleProfessionals "The Place to Find Qualified Professionals with Disabilities". The AbleProfessionals name comes from a line of more than a dozen office tools called The AbleOffice which are marketed worldwide by CRT, Inc. These tools are designed to assist people with disabilities to function productively within a standard office environment. AbleOffice products feature universal design and work for both the disabled and general populations. They are complementary office tools that give people with disabilities the abilities to access files, store and use books and manuals, open mail, use the telephone, and access computers and printers. Products are modular and fit neatly in with modular office decor. In the past three years, more than 90 AbleOffice systems have been installed at locations in 24 states in the US as well as three foreign countries.

\section{'One Stop Shopping' Approach to Recruiting, Employing and Accommodating}

When an employer confronts the problems with computer technology, office equipment and architectural barriers and considers bringing a person with a disability into their company anyway there are a thousand unanswered questions. Questions like, "can we cope with the accommodations this new employee will require," "can we afford the accommodations for this person;" and "will this person fit in at our company, will they understand and accept our corporate culture?" Questions on another level include, "what if this person doesn't work out?", "will that failure be our fault?", "will we end up with a legal problem?" Traditionally employers have to seek answers to these questions from different experts in various locations. What happens is that employers' good intentions are penalized with having to spend additional time and money trying to get help in their endeavor to hire the person needing the accommodation.

The AbleProfessionals solution is to offer a business service team that provides 'one- stop shopping' expertise to:

- locate, recruit and coordinate employment of quality, qualified people with disabilities and

- screen candidate clients for qualifications, quality and suitability and

- provide complete, cost effective accommodation service over the long term. 


\section{AbleProfessionals Provide Continuing Technological and Accommodation Support to Their Clients over the Long Term}

Part of the original contract agreement AP makes with the employer will concern long term technical and accommodation support for any clients that are hired under the agreement. This clause is intended to ensure that once the client is successfully employed that they continue in their employment despite changes in their physical condition. With changes in their physical abilities AP would bring back the experts in accommodation from CRT to re-adjust or re-design any accommodation that the employee needed as a result of a change in the employee's abilities.

The other part of the long term support has to do with new capabilities in office equipment or computer technology. AP would be responsible for keeping its clients continuously informed about advancements in technology. When one of the advancements solved a technical problem for an AP client, AP would take the responsibility for acquiring and installing that equipment. An example of this with regard to the computer systems analyst with the vision impairment concerns the limitation of the screen reading software to handle Windows based operating systems and applications. Currently the screen reading software does not accommodate Windows. When the technology breaks through and Windows can be handled by screen reading software, or in some other fashion can be translated into sound AP would take the initiative and ensure its clients with vision impairments got the word and were able to take advantage of the new technology.

\section{Conclusions}

AbleProfessionals is founded on the premise that the employment potential of people with disabilities increases with the growing incorporation of computer technology into the business world. All that need be done is to bring the people with the technical know-how together with the employers who have the vacancies and the people with disabilities who have the education, experience and the need for the job and the accommodation they require to do it - AbleProfessionals will take care of the rest. This arrangement permits each participant to leverage the competencies of the other two parties in this agreement. Each party is able to avoid the costly option of vertical integration. In other words, the corporate human resource staffs of the organizations that sign up with AbleProfessionals are liberated from the tasks of recruiting, placing and accommodating people with disabilities. They can out source the task to AbleProfessionals who will complete it at a lower cost and higher value-added than the buying corporation can. The cost of the technology may be lower because AP will be coordinating the acquisition of the computer equipment working the best deals and substituting less expensive technology where possible. Further, AbleProfessionals offers a flexible menu of services to its corporate clients. Corporations can choose the services they want to contract for. This flexibility is useful if a business wants to contract for AP services for an existing employee, who because of changing circumstances needs a different piece of equipment, or who needs a different accommodation because of changing business practices on the part of the corporation. AbleProfessionals would assist in researching, acquiring, installing and training the employee to use their new equipment. 